\title{
Granulomatous inflammation in lymph nodes draining cancer: significant association or just coincidence- Diagnostic dilemma
}

\author{
Rakesh Kumar J. Ajmera ${ }^{1, *}$, Rohini Shewale ${ }^{2}$, Hemant Kokandakar ${ }^{3}$ \\ ${ }^{\mathbf{1}}$ Assistant Professor, ${ }^{2}$ House Officer, ${ }^{3}$ Professor, Dept. of Pathology, Government Cancer Hospital, Aurangabad, Maharashtra, \\ India
}

*Corresponding Author:

Emai: drrakeshajmera@yahoo.in

\begin{abstract}
Causes of granulomatous inflammation are broadly divided into infective and non infective. Infective causes Includes myocbacterial, fungal or parasitic origin. Non infective includes: Foreign body granuloma, sarcoidosis, crohn's disease. Granuloma formation seen in cancer patients may be due to previous chemotherapy or radiation therapy. However in many cases mechanism in granuloma formation and its therapeutic outcome in cancer patients remains unknown. Careful scrutiny in all cases is required where coexisting malignancy was suspected along with granuloma formation in lymph nodes draining cancers. Exact cause of granuloma formation in lymph nodes draining cancer remains unknown however immunological reaction to tumour antigens is proposed theory. These granuloma may represent impending metastasis or already existing metastasis in patients who received neoadjuvant chemoradiation.
\end{abstract}

Keywords: Granuloma, Lymph nodes, Metastasis, Chemoradiation.

\section{Introduction}

Granulomatous inflammation is defined as a chronic inflammatory reaction characterized by microscopic aggregation of activated macrophages with epitheloid appearance. ${ }^{1}$ These may or may not be associated with mononuclear cells, multinucleate giant cells and necrosis.

Diagnosing granuloma on routine $\mathrm{H}$ and $\mathrm{E}$ staining is usually straightforward. However to look for causes of granulomatous inflammation requires further evaluation /tests. Causes of granulomatous inflammation are broadly divided into infective and non infective. Granulomas can be necrotizing or non necrotizing.

Infective: Includes myocbacterial, fungal or parasitic origin. Non infective includes: Foreign body granuloma, sarcoidosis, crohn's disease etc.

Apart from these common causes granuloma formation may be seen in neoplastic disorders. These include as a part of primary cancer or granuloma in draining lymph nodes of various cancers. This granuloma may arise due to preexisting infective illness like mycobacterial or others or due to immunosuppresion in cancer patients leading to infection.

In developing country like India tuberculous etiology should be considered or ruled out before considering other causes.

Granuloma formation seen in cancer patients may be due to previous chemotherapy or radiation therapy. However in many cases mechanism in granuloma formation and its therapeutic outcome in cancer patients remain unknown.

It has been observed in many malignancies e.g. breast carcinoma, gastric, colonic and laryngeal cancer $^{2}$

\section{Materials and Methods}

Present study was carried out in tertiary care cancer hospital. All cases of Granuloma formation seen in draining lymph nodes or in primary tumor were included in study. Lymph node dissection done as a part of management of cancer patients in all cases were studied .Common surgeries included neck dissection performed for head neck cancers. Study included all consecutive surgeries performed in cancer patients during one and half year. However known diagnosed cases of tuberculosis with cancer were excluded. After gross examination to look for obvious caseation; sections were processed as a routine processing and studied with $\mathrm{H}$ and E staining.

Z. N. staining for mycobacteria was carried as a routine in all cases showing granuloma formation.

Detailed history regarding past history of TB, chemotherapy or radiation received by patients was recorded. Granuloma formation particularly caseating granuloma were informed to treating clinician apart from mentioning it in pathology report. Careful scrutiny and Deep cut sections were studied in all cases where coexisting malignancy was suspected along with granuloma formation to avoid under diagnosis of metastatic disease. Since tissue were formalin fixed primarily to diagnose cancer, ancillary techniques like Gene expert which requires fresh tissue in normal saline were not carried out to confirm Tuberculous etiology of granuloma. However TB-PCR study was carried out in one case.

\section{Observation and Results}

Of all consecutive lymph node dissection done for cancer patients during one half year, 27 cases showed granulomatous inflammation in lymph nodes. 


\section{Graph 1: Agewise distribution of cases}

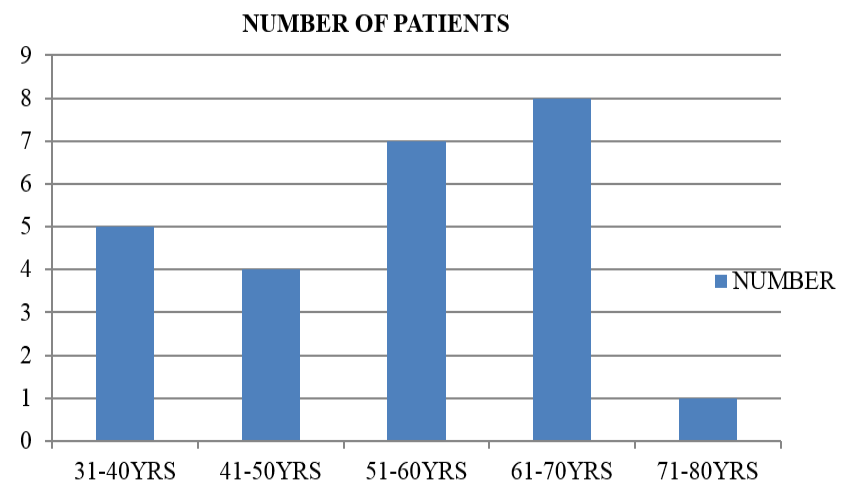

Of these cases 16 cases were of neck dissection performed for head and neck cancer.

Squamous cell carcinoma was most common malignancy seen in association with granulomatous inflammation in lymph nodes. This finding maybe attributed to common head neck surgeries performed in our institute. In five cases, granulomas were associated with necrosis i.e. necrotizing granulomatous inflammation. In other twenty two cases granulomas were non caseating granuloma.

Z.N. stain for detection of mycobacteria carried out in all cases was negative for Acid Fast Bacilli. TBPCR done in one case showed negative result.

No obvious fungal or parasitic elements were seen on routine $\mathrm{H}$ and $\mathrm{E}$ staining. In three cases surgery was done post neoadjuvant chemotherapy of these two case showed only granuloma formation with no residual viable tumour.

one case was of multiple paraganglioma with neck dissection showing granuloma formation.

Among five cases of breast lump one case was of benign phyllodes tumour with axillary nodes showing granuloma formation.

Also one case of diffuse large B cell Lymphoma post 6 cycles of chemotherapy showed granuloma formation in follow up biopsy.

Of these all cases total two cases showed presence of granuloma and metastatic disease in lymph nodes simultaneously.

\section{Graph 2: Distribution of malignancies with granuloma}

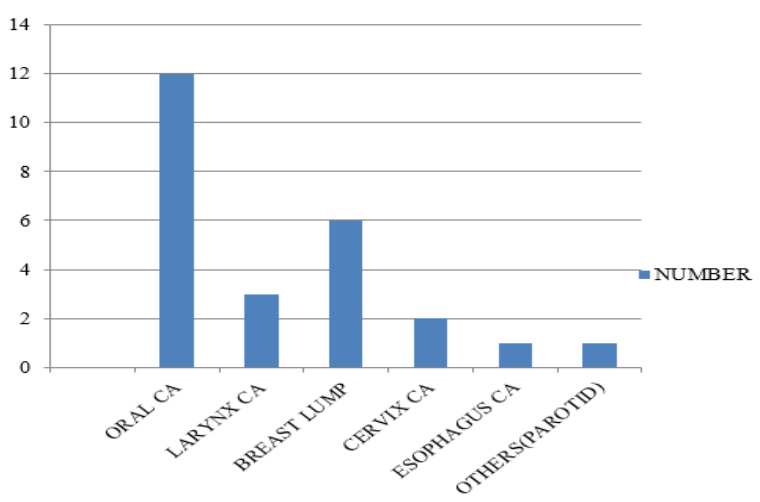

Summary of patients with granulomatous reaction: Data presented as number

\begin{tabular}{|l|l|}
\hline No. of patients in whom ziehl neelsen stain done & All twenty seven cases \\
\hline No. of patients in whom ziehl neelsen stain positive & None \\
\hline No. of patients in whom tb-pcr done & One patient [Negative result] \\
\hline Neoadjuvant chemo/radiation received & Two cases \\
\hline $\begin{array}{l}\text { Only granulomatous reaction with no residual viable } \\
\text { tumour }\end{array}$ & Two cases \\
\hline $\begin{array}{l}\text { Number of cases with simultaneous granuloma and } \\
\text { sceonderies in lymph nodes }\end{array}$ & Two cases \\
\hline
\end{tabular}

Various types of granulomatous inflammation were seen in lymph nodes draining cancer which included:
1] Confluent or focal granulomas; 2] Granuloma with fibrinoid necrosis; 3] caseating granuloma; 4] sarcoid 
like granuloma -composed of epitheloid cells and lymphocytes.

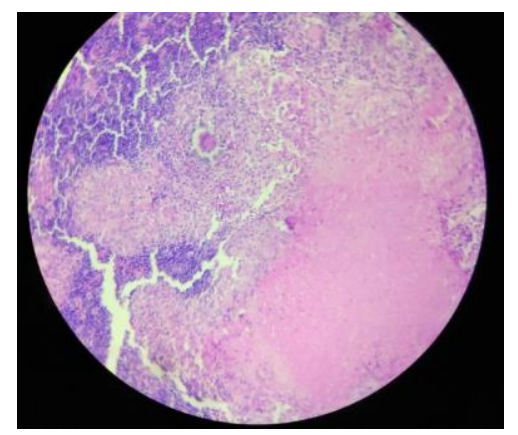

Fig. 1: Necrotic granulomatous inflammation with giant cells

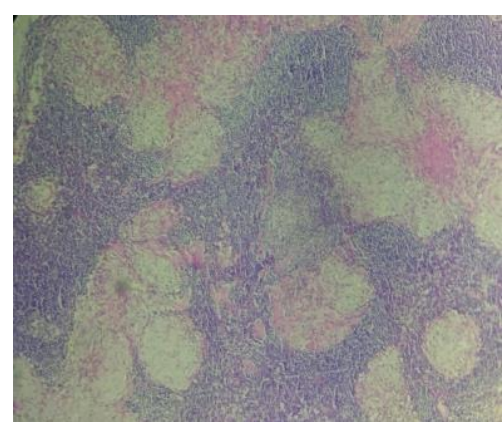

Fig. 2: Granuloma with fibrinoid necrosis and amyloid like deposition

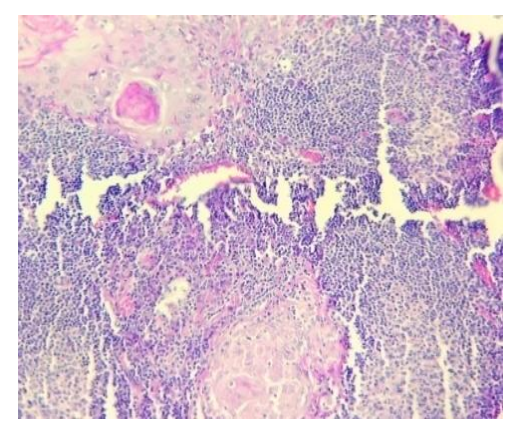

Fig. 3: Keratin granuloma with seconderies of squamous cell carcinoma

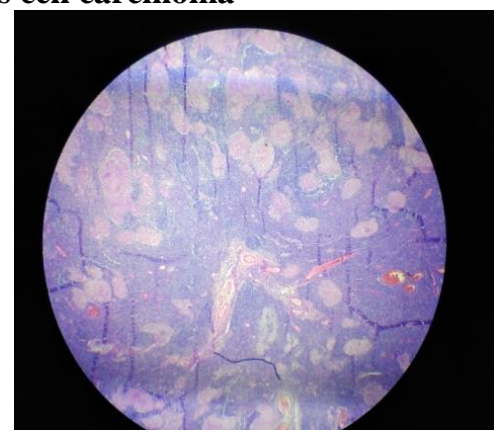

Fig. 4: Scanner view of Confluent non caseating granuloma

\section{Discussion}

Possible causes of granulomatous inflammation in cancer draining lymph nodes includes:
1. Keratin granulomas in squamous cell carcinoma.

2. Sarcoid like reaction to tumor antigens.

3. Therapy related granuloma formation. (chemoradiation)

4. Associated systemic illness like tuberculosis.

Although Negative Z. N. stain does not rule out tuberculosis, prior history, clinical and radiological findings are helpful. There is possibility of potentially exacerbating tuberculosis illness due to chemotherapy and immune suppression further ancillary studies are recommended to rule out tuberculosis illness even if z.n. stain is negative.

Careful scrutiny and Deep cut sections were studied in all cases where coexisting malignancy was suspected along with granuloma formation to avoid under diagnosis of metastatic disease. this is particularly important since in study carried out by Coyne et $\mathrm{al}^{3}$ showed presence of microinvasive breast carcinoma with granulomatous inflammation which required close scrutiny since invasive component measuring $<1 \mathrm{~mm}$. This is particularly important to avoid consequences of missed/underdiagnosis malignancies in patients with granuloma and eventual outcome of patient. In our study case of Ca esophagus showed only small microscopic focus of residual tumor post NACT along with granuloma formation.

A careful search under the microscope may reveal the presence of malignant cells. However, sometimes it may be difficult to identify the tumour cells in these nodes with haematoxylin and eosin stain alone and immunohistochemistry is required(Syrjanen, $1981)^{4}$,Therefore apart from evaluating for causes of granuloma IHC studies done for malignancy cases is also required.In our study case of Lymphoma with granulomatous reaction IHC study was suggested.

Granuloma-associated conditions constitute a very heterogeneousroup of diseases it is not possible to suggest a standard set of investigations which will, eventually, leadto the correct diagnosis. However, a number of possibilities should be considered in a systematic way. Firstly, anamnestic information on sarcoidosis and granuloma associated Diseases should be obtained. As regards sarcoidosis, not all patients are aware of this diagnosis, and it may be necessary to review patient records as well as chest x-rays from previous hospital admissions. Secondly, a thorough clinical examination should focus on findings relevant to granuloma-associated disease. Thirdly, traditional histomorphological evaluation of granulomatous lesions Biopsy materials should be supplemented with immunohistological studies. ${ }^{5}$

Present study showed one case of benign phylloides tumour with granuloma formation in axillary lymph nodes, similar findings were seen in case reported by Prem singh et $\mathrm{al}^{6}$ however in their study $\mathrm{Z}$. N. stain revealed presence of Acid fast Bacilli.

Among squamous malignancies showing granuloma formation most common malignancy site 
was oral cavity followed by larynx, Uterine cervix, this finding was in concordance with study carried out by HOMA SAFAII ${ }^{7}$ et al in which they studied keratin granuloma in irradiated squmous cell carcinoma.

Knowledge of these granuloma is essential particularly for primary upfront diagnosis done on biopsy or FNAC, and to rule out focus of malignancy. presence of these granuloma without obvious malignancy warrants further evaluation including repeat biopsy as these specimens may misdiagnose cancer as TB with false negative results. ${ }^{8}$

\section{Comparision of findings of Z.N.stain among different studies}

\begin{tabular}{|l|l|}
\hline 1. ${\text { Chabra et } \mathrm{al}^{9}}^{9}$ & Three out of seven cases showed ZN positive for acid fast bacilli \\
\hline 2. Premsingh et $\mathrm{al}^{6}$ & One case showed ZN positivity for acid fast bacilli. \\
\hline 3. Bushra siddiqui et $\mathrm{al}^{10}$ & One case showed Z.N.negativity. \\
\hline 4. Minas khurram et al ${ }^{11}$ & All twenty two cases were Z.N. negative. \\
\hline 5. Present study & All twenty seven cases were Z.N. negative. \\
\hline
\end{tabular}

So findings of our study is similar to observations of Minas khurram et $\mathrm{al}^{11}$ which also showed $\mathrm{ZN}$ stain negativity in all cases studied. However their study recommended PCR Based assays for diagnosis of TB, which was carried out in only case in present study and revealed negative result.

This is only study to best of our knowledge which included all spectrums of malignancies i.e. carcinoma, lymphoma, paraganglioma, phylloides tumour were included with granuloma formation.

\section{Conclusion}

Granulomatous inflammation in draining lymph nodes for cancer patients is not uncommon finding.

Close scrutiny and further evaluation of these patients is necessary to rule out tuberculous etiology particularly in developing countries where TB is rampant. However still interpretation of granulomatous inflammation with neoplasia remains difficult. Even when all relevant information is obtained, the interpretation of granulomatous lesions in neoplasia will remain difficult. Thus, it will quite often be necessary to combine traditional methods of clinical evaluation and immunohistological studies with prolonged follow-up in order to establish the true nature of a granuloma-associated condition.so it is emphasized that if histopathology examination reveals granuloma formation it require evaluation not only for cause of granuloma but also for concomitant malignancy like Immunohistochemistry study to avoid false negative results.

However diagnosis, prognosis, management of these cancer patients with granulomatous reaction requires further research.

\section{References}

1. Cotran, Ramzi S.; Kumar, Vinay; Fausto, Nelson; Nelso Fausto; Robbins, Stanley L.; Abbas, Abul K.
(2005). Robbins and Cotran pathologic basis of disease (7th ed.). St. Louis, Mo: Elsevier Saunders. pp. 82. ISBN 0-7216-0187-1.

2. Ophir D, Nissim F, Marshak G. Granulomatous reaction in lymph nodes draining laryngeal carcinoma. Arch Otolaryngol Head Neck Surg 1985;3:214-17.

3. Coyne JD, Haboubi NY. Micro-invasive breast carcinoma with granulomatous stromal response. Histopathol 1992;20:184-85.

4. Syrjanen KJ. Epithelioid cell granulomas in the lymph nodesdraining human cancer: ultrastructural findings of a breast cancercase. Diagn Histopathol 1981;291-94.

5. Hans Brincker. Interpretation of Granulomatous Lesions in Malignancy. Acta Oncologica 1992;31(1):85-89, DOI: 10.3109/02841869209088273.

6. Prem Singh, Jyoti Bala, Deeba Mushtaq, Mayank Madan. Phyllodes tumour of the breast associated with concomitant tuberculous and sarcoid like lesions in the axillary lymph nodes: a case report. J Clin Diagnostic Res [serial online] 2011;5:610-12.

7. Homa Safaii and Henry A. Azar Keratin granulomas in irradiated squamous cell carcinomas of various sites. Cancer Res 1966;26:500-8.

8. Dagaonkar RS, Choong CV, Asmat AB, et al. J Clin Pathol 2017;70:337-41.

9. Chhabra S, Mohan H, Bal A. Granulomas in association with neoplasm: A reaction or a different primary process? J Postgrad Med [serial online] 2009;55:234-36.

10. Siddiqui B, Habib Faridi S, Maheshwari V, Aslam M, Akhtar K. Granulomatous respose with breast cancer in a developing country :A diagnostic dilemma. Iran J Pathol 2016;11(2):171-75.

11. Khurram M, Tariq M, Shahid P. Breast cancer with associated granulomatous axillary lymphadenitis: A diagnostic and clinical dilemma in regions with high prevalence of tuberculosis. Pathol Res Pract 2007;203(10):699-704.[PubMed]

How to cite this article: Ajmera R., Shewale R., Kokandakar H. Granulomatous inflammation in lymph nodes draining cancer: significant association or just coincidence- Diagnostic dilemma. J Diagn Pathol Oncol 2018;3(4):295-98. 